\title{
Mexiletine Hydrochloride
}

National Cancer Institute

\section{Source}

National Cancer Institute. Mexiletine Hydrochloride. NCI Thesaurus. Code C652.

The hydrochloride salt form of mexiletine, a local anesthetic and antiarrhythmic (Class IB) agent structurally related to lidocaine. Mexiletine exerts its antiarrhythmic effect by inhibiting the inward sodium current in cardiac cells, thereby reducing the rate of rise of the cardiac action potential (phase 0 ) and decreases automaticity in the Purkinje fibers. This slows nerve impulses in the heart and stabilizes the heartbeat. Mexiletine's anesthetic activity is due to its ability to block sodium influx in peripheral nerves, thereby reducing the rate and intensity of pain impulses reaching the central nervous system. 